\title{
Gestión Pedagógica Directivo y Proyectos Educativos Productivos en las instituciones educativas del distrito de Azángaro Provincia de Yauyos - Lima, 2020
}

\author{
Directive Pedagogical Management and Productive \\ Educational Projects in the educational institutions of the \\ district of Azángaro Province of Yauyos - Lima, 2020
}

Edith Kredis Flores Alfaro ${ }^{1}$ (D) Abelardo Rodolfo Campana Concha ${ }^{1}$

${ }^{1}$ Universidad Nacional Mayor de San Marcos. Lima, Perú

Recibido: 19/04/2021

Revisado: 18/05/2021

Aceptado: 08/05/2021

Publicado: 31/ 06 / 2021

\section{RESUMEN}

El objetivo de la investigación es determinar la relación entre la gestión pedagógica directiva y los Proyectos Educativos Productivos en las Instituciones Educativas del distrito de Azángaro provincia de Yauyos -Lima, 2020. Para su desarrollo se consideró la investigación correlacional en la que se analizarán el vínculo estadístico entre dos variables, aplicándose un cuestionario para cada variable aplicado a una muestra de 88 docentes, cuyos datos recolectados fueron procesados mediante el programa estadístico SPSS, además se empleó Rho de Spearman con un margen de error 5\%. Los resultados hallaron un nivel de correlación de 0,904 evidenciando relación entre gestión pedagógica directiva y proyectos educativos productivos, debido a los resultados mayoritarios de la gestión pedagógico directivo considerado de indiferente por el $73,9 \%$, lo cual está vinculado a los proyectos educativos productivos visto por el $71,6 \%$ con una frecuencia de a veces. Del mismo modo, se confirmó la relación de la primera variable con las dimensiones pertinencia, objetividad, compromiso y creatividad. Por ello, se concluyó que una gestión intermedia de los directivos dentro de la institución limita el interés institucional (docentes, estudiantes y padres de familia), para el desarrollo efectivo de los proyectos educativos. Palabras clave: Gestión pedagógico, proyecto productivo, objetividad, compromiso, creatividad.

\begin{abstract}
The objective of the research is to determine the relationship between the directive pedagogical management and the Productive Educational Projects in the Educational Institutions of the district of Azángaro province of Yauyos - Lima, 2020. For its development, the correlational research was considered in which the link will be analyzed statistical between two variables, applying a questionnaire for each variable applied to a sample of 88 teachers, whose data collected were processed through the statistical program SPSS, in addition, Spearman's Rho was
\end{abstract}


used with a margin of error of $5 \%$. The results found a correlation level of 0,904 showing a relationship between directive pedagogical management and productive educational projects, due to the majority results of directive pedagogical management considered indifferent by $73.9 \%$, which is linked to productive educational projects seen by the $71.6 \%$ with a frequency of sometimes. In the same way, the relationship of the first variable with the relevance, objectivity, commitment and creativity dimensions was confirmed. Therefore, it was concluded that an intermediate management of the directors within the institution limits the institutional interest (teachers, students and parents), for the effective development of educational projects. Keywords: Pedagogical management, productive project, objectivity, commitment, creativity.

\section{INTRODUCCIÓN}

Hoy en día, el currículo está orientado a fomentar el conocimiento en los educandos, al desarrollo en los aspectos físico, afectivo y cognitivo. Este currículo está direccionado a desarrollar aprendizajes en los educandos promoviendo el conocimiento. Si bien están las competencias orientadas a formar estudiantes críticos, reflexivos y creativos, debemos no descuidar las capacidades en el área ocupacional, que es allí donde los estudiantes aprenden cosas prácticas que le ayudarán en la acción del quehacer cotidiano, mucho más aún si son de zonas rurales, o de serranía donde tienen en muchos casos limitado acceso a la educación superior.

En las zonas altas andinas y en las rurales de nuestro país que en su mayor parte comprenden nuestro territorio patrio las familias necesitan el aprendizaje de técnicas priorizando trabajos propios del lugar, para abastecer su economía.

Es aquí donde los directores de las diversas instituciones educativas deben promover proyectos basados en la formación laboral de los educandos, proyectos que sean productivos y que solventen la economía familiar; y que los estudiantes al aprender las diversas acciones ocupacionales, aporten si no tienen acceso a la educación superior, al progreso de sus familias.
Dirigir una institución educativa es acción del director; y gestionar proyectos productivos que influyan en hacerlos competentes a los estudiantes para la vida y el trabajo es tarea fundamental del líder pedagógico.

Es importante entender que la gestión pedagógica directiva se describe como "el proceso por el cual se llegarán a organizar las instituciones educativas para lograr formar individuos de bien y otros colectivos" (Sañudo, 2015).

Por otro lado, Baltar (2015) menciona que el proyecto educativo productivo es un conjunto de ideas, escritos, gráficas, etc., direccionados a buscar soluciones razonadas frente a un problema sin importancia el grado de complejidad, es decir, debe resolver para obtener la satisfacción de un alcance productivo.

Este estudio se apoyó de estudios anteriores, como los de Cortés (2020, p.25) encuentra que la aplicación de este proyecto ayuda al estudiante a desarrollar un pensamiento analítico y reflexivo, con competencias enfocadas en el liderazgo, responsabilidad ambiental, trabajo colectivo, solidario y transformador, así como formar una identidad en miras hacia el futuro.

Por su parte, Damas y Oliva (2017) establecen la relación de las variables señalan 
do que la eficiencia de la gestión educativa consigue la satisfacción estudiantil. Por ello, sugiere a los directores de las instituciones efectuar mayor participación docente, y administrativa, con el fin de desarrollar trabajos más coordinados para el establecimiento de funciones y trabajo democrático en busca de una mejor calidad educativa.

Por tanto, el objetivo de este estudio fue determinar la relación entre la gestión pedagógica directiva y el desarrollo de proyectos Educativos productivos en las Instituciones Educativas.

\section{MATERIALES Y MÉTODO}

Es un estudio correlacional con enfoque cuantitativo, nivel explicativo, ex post facto, puesto que intenta analizar y evaluar la relación entre las dos variables de estudio, 88 representan la muestra. Se les aplicó dos cuestionarios que corresponde a la variable 1 y 2 , cuya información fue procesada mediante el programa estadístico SPSS.

\section{RESULTADOS Y DISCUSIÓN}

Tabla 1. Frecuencias Gestión Pedagógica Directivo

\begin{tabular}{llrr}
\hline & & Frecuencia & Porcentaje \\
\hline \multirow{4}{*}{ Válidos } & Poco adecuado & 6 & 6,8 \\
& Indiferente & 65 & 73,9 \\
\cline { 2 - 4 } & Adecuado & 17 & 19,3 \\
\cline { 2 - 4 } & Total & 88 & 100,0 \\
\hline \multicolumn{3}{c}{ Fuente. Elaboración propia }
\end{tabular}

En la tabla 1, el 73,9\% de la muestra considera estar indiferente a la gestión pedagógica directivo, el 19,3\% lo refieren de adecuado y el $6,8 \%$ de poco adecuado, por lo cual se evidencia relativa insatisfacción del docente.
Tabla 2. Frecuencias proyectos educativos productivos

\begin{tabular}{llrr}
\hline & & Frecuencia & Porcentaje \\
\hline \multirow{4}{*}{ Válidos } & Casi nunca & 6 & 6,8 \\
& A veces & 63 & 71,6 \\
& Casi siempre & 19 & 21,6 \\
\cline { 2 - 4 } & Total & 88 & 100,0 \\
\hline
\end{tabular}

Fuente. Elaboración propia

En la tabla 2, el 71,6\% de la muestra considera que solo a veces que aplican proyectos educativos productivos, el $21.6 \%$ refiere que casi siempre y el $6.8 \%$ casi nunca, es decir, los docentes consideran que se desarrolla de forma irregular. Luego de estos resultados, se procesó el nivel de relación de las variables expuestas en la hipótesis general, que a continuación se detalla:

HGA. La Gestión pedagógica directivo se asocia directamente con Proyectos educativos productivos en las instituciones educativas. HGO. La Gestión pedagógica directivo no se asocia directamente con Proyectos educativos productivos en las instituciones educativas.

Tabla 3. Correlación gestión pedagógica directivo y proyectos educativos productivos.

\begin{tabular}{lllr}
\hline & Correlación & \multicolumn{1}{c}{$\begin{array}{c}\text { Proyectos } \\
\text { educativos } \\
\text { productivos }\end{array}$} \\
\hline \multirow{2}{*}{$\begin{array}{l}\text { Rho de } \\
\text { Spearman }\end{array}$} & $\begin{array}{l}\text { Gestión } \\
\text { pedagógica } \\
\text { directivo }\end{array}$ & $\begin{array}{l}\text { Coeficiente de } \\
\text { correlación }\end{array}$ &, 904 \\
\cline { 3 - 4 } & Sig. (bilateral) &, 000 \\
\hline
\end{tabular}

Fuente. Elaboración propia

En la tabla 3, se halló una relación muy buena $(\mathrm{Rho}=, 904)$ con un $\mathrm{p}=0,000$ entre las variables 
de estudio, lo que permite aceptar el planteamiento de la hipótesis alterna, además se confirma que la acción indiferente en la pedagógica de los directores, permite que los Proyectos Educativos Productivos se apliquen a veces. Por otro lado, también se plantearon las hipótesis específicas, que a continuación se muestran luego de su aplicación estadística:

Ha1. La Gestión pedagógica directivo se asocia directamente conla dimensión pertinencia en las instituciones educativas.

Ho. La Gestión pedagógica directivo se asocia directamente con la dimensión pertinencia en las instituciones educativas.

Tabla 4. Correlación de la hipótesis específica 1

\begin{tabular}{lllr}
\hline & \multicolumn{2}{c}{ Correlación } & Pertinencia \\
\hline \multirow{2}{*}{$\begin{array}{l}\text { Rho de } \\
\text { Spearman }\end{array}$} & $\begin{array}{l}\text { Gestión } \\
\text { pedagógica } \\
\text { directivo }\end{array}$ & $\begin{array}{l}\text { Coeficiente de } \\
\text { correlación }\end{array}$ &, $671^{* *}$ \\
\cline { 3 - 4 } & Sig. (bilateral) &, 000 \\
\cline { 3 - 4 } & & $\mathrm{N}$ & 88 \\
\hline
\end{tabular}

Fuente. Elaboración propia

En la tabla 4, se confirma una buena relación de Rho $=, 671$ y un $p=0,000$ cuyos datos estadísticos confirman el planteamiento de la hipótesis alterna, además confirma que la acción indiferente en la gestión pedagógica de los directores, permite que la pertinencia se aplique a veces.

Ha2. La Gestión pedagógica directivo se asocia directamente con la dimensión objetividad en las instituciones educativas.

Ho.La Gestión pedagógica directivonose asocia directamente con la dimensión objetividad en las instituciones educativas.
Tabla 5. Correlación de la hipótesis específica 2

\begin{tabular}{lllr}
\hline & \multicolumn{2}{c}{ Correlaciones } & Objetividad \\
\hline \multirow{3}{*}{$\begin{array}{l}\text { Rho de } \\
\text { Spearman }\end{array}$} & $\begin{array}{l}\text { Gestión } \\
\text { pedagógica } \\
\text { directivo }\end{array}$ & $\begin{array}{l}\text { Coeficiente de } \\
\text { correlación }\end{array}$ &, $702^{*}$ \\
\cline { 3 - 4 } & Sig. (bilateral) & N & 800 \\
\hline
\end{tabular}

Fuente. Elaboración propia

En la tabla 5, la significancia de 0.00 y el coeficiente 0,702 explican la relación buena entre la variable y la dimensión, lo cual permite aceptar la Ha., además que comprende que la acción indiferente en la gestión pedagógica de los directores, permite que la objetividad se aplique a veces.

Ha3. La Gestión pedagógica directivo se asocia directamente con la dimensión compromiso en las instituciones educativas.

Ho. La Gestión pedagógica directivo se asocia directamente con la dimensión compromiso en las instituciones educativas.

Tabla 6. Correlación de la hipótesis específica 3

\begin{tabular}{lllr}
\hline & \multicolumn{2}{c}{ Correlaciones } & Compromiso \\
\hline \multirow{2}{*}{$\begin{array}{l}\text { Rho de } \\
\text { Spearman }\end{array}$} & $\begin{array}{l}\text { Gestión } \\
\text { pedagógica } \\
\text { directivo }\end{array}$ & $\begin{array}{l}\text { Coeficiente de } \\
\text { correlación }\end{array}$ & \begin{tabular}{l} 
Sig. (bilateral) \\
\cline { 3 - 4 }
\end{tabular} \\
\hline
\end{tabular}

Fuente. Elaboración propia

En la tabla 6, se comprueba una buena relación de $R$ ho $=, 658$ y un $p=0,000$ entre la variable $y$ la dimensión que según el procedimiento estadístico permite aceptar de la hipótesis alterna, asimismo se entiende que la acción indiferen 
te en la gestión pedagógica de los directores, permite que el compromiso se aplique a veces.

Ha4. La Gestión pedagógica directivo se asocia directamente con la dimensión creatividad en las instituciones educativas.

Ho. La Gestión pedagógica directivo no se asocia directamente con la dimensión creatividad en las instituciones educativas.

Tabla 7. Correlación entre gestión pedagógica y la creatividad

\begin{tabular}{lllr}
\hline & \multicolumn{2}{c}{ Correlación } & Creatividad \\
\hline \multirow{3}{*}{$\begin{array}{l}\text { Rho de } \\
\text { Spearman }\end{array}$} & $\begin{array}{l}\text { Gestión } \\
\text { pedagógica } \\
\text { directivo }\end{array}$ & $\begin{array}{l}\text { Coeficiente de } \\
\text { correlación }\end{array}$ &, $593^{* *}$ \\
\cline { 3 - 4 } & Sig. (bilateral) &, 000 \\
\cline { 3 - 4 } & $\mathrm{N}$ & 88 \\
\hline
\end{tabular}

Fuente. Elaboración propia

En la tabla 7, se confirma la existencia de una relación moderada $(\mathrm{Rho}=, 593)$ con un $\mathrm{p}=$ 0,000 entre la variable y dimensión, lo que confirma el planteamiento de la hipótesis alterna, por otro lado, se entiende que la acción indiferente en la pedagógica de los directores, permite que la creatividad se aplique a veces. En base al procedimiento estadístico entre la variable $\mathrm{X}$ e $\mathrm{Y}$, la hipótesis general estableció una relación muy buena, debido a los resultados mayoritarios de la gestión pedagógico directivo considerado de indiferente por el $73,9 \%$, lo cual está vinculado a los proyectos educativos productivos visto por el $71,6 \%$ con una frecuencia de a veces. Con ello, se evidencia la falta de mayores adecuaciones en este tipo de gestión por parte de los directores para incrementar la frecuencia en la cual se plantean proyectos en beneficio de la educación.
En la propuesta de hipótesis estadística 2 se comprobó la relación buena $(\mathrm{Rho}=, 671)$, cuya mayoría considera que la gestión pedagógica directiva es indiferente por el $62,5 \%$, lo cual encontró relación en las acciones de pertinencia calificado por el $63.6 \%$ con una frecuencia de a veces. En otras palabras, la satisfacción media en la forma como se desarrolla este tipo de gestión disminuye las posibilidades de brindar mayor énfasis en la búsqueda de soluciones en los problemas de aprendizaje, la promoción de logros de aprendizaje y la concientización de la diversidad de su entorno social. En la HE2 identificó una relación buena (Rho $=.0,702)$, en donde la mayoría representado por el $62,5 \%$ califica la gestión pedagógica directiva es indiferente, lo cual está involucrado a los resultados de la objetividad aplicado a veces según el 59,1\%. Es decir, la satisfacción intermedia en la forma como se manifiesta esta gestión disminuye las intenciones de que el proyecto se ejecute satisfactoriamente, se enfoque en resolver problemas de aprendizaje y se ejecute productivamente los temas propuestos.

El planteamiento hipotético 3 halló una buena relación de $\mathrm{Rho}=, 658$ debido a la relación estadística entre la gestión pedagógica directiva y el compromiso, en donde la mayoría de los docentes consideraron que la dimensión es indiferente $(73,9 \%)$, en tanto que la dimensión es categorizada de a veces (52,3\%). En resumen, la forma en la que los docentes aprecian esta gestión disminuye la proactividad de los estudiantes y su participación con nuevas ideas para la ejecución de proyectos educativos. En la hipótesis específica 4 se confirmó una relación moderada de $\mathrm{Rho}=$,593) debido a la perspectiva de la muestra quien en su mayoría $(73,9 \%)$ considera que la gestión pedagógica directiva es indiferente, mientras que la crea- 
tividad es vista como a veces $(51,1 \%)$. Es decir, los docentes consideran que la gestión liderada por los directivos está limitando el desarrollo de la originalidad y el tiempo de aplicación del proyecto, así como la iniciativa estudiantil.

Los resultados obtenidos tienen relativa similitud con los de Rojas y González (2015) en cuanto a la preocupación en la gestión institucional, debido a que el investigador considera que es necesario un análisis del contexto educativo para potenciar los proyectos educativos, sin embargo es importante agregar que el director, al ser la cabeza del equipo de trabajo, debe ser quien impulse ello, sin embargo con las actitudes indiferentes a la gestión y su compromiso en los Proyectos Educativos Productivos categorizado como a veces, no se evidencia un interés claro en buscar mejores resultados.

Mendoza (2013) en su investigación concluye que siempre o casi siempre se consiguen aprendizajes significativos con la aplicación de proyectos productivos, en tanto que nuestro estudio alerta que sucede a veces, pero no por la efectividad sino por el compromiso de los directores quienes gestionan todos los elementos necesarios para buenos resultados.

\section{CONCLUSIONES}

La investigación confirmó que una gestión intermedia de los directivos dentro de la institución limita el interés institucional (docentes, estudiantes y padres de familia), para el desarrollo efectivo de los proyectos educativos.

Del mismo modo, confirma una posición intermedia en las acciones de preocupación por el personal, instrucción, monitoreo y acciones comunitarias solo lleva a un avance con la misma categorización en los proyectos educativos en términos de soluciones a los problemas de aprendizaje, la promoción de logros de aprendizaje y la concientización de la diversidad de su entorno social.

En tanto que la gestión pedagógica directiva (indiferente) está conectada estadísticamente a la objetividad (a veces), que en otras palabras hace referencia a que este tipo de gestión apunta acciones con una frecuencia irregular impidiendo que los proyectos educativos se desarrollen regularmente con un enfoque resolutivo en los problemas de aprendizaje y ejecución productiva de los temas propuestos.

Del mismo modo, la gestión pedagógico directivo apunta acciones con una frecuencia irregular, lo cual hace que los proyectos educativos se desarrollen con una insatisfacción intermedia en cuanto a la proactividad de los estudiantes y su interés participativo mediante nuevas ideas.

Para finalizar, evidenciamos que la gestión pedagógica accionado de forma irregular conlleva a que los proyectos educativos se desarrollen con satisfacción intermedia del proyecto educativo en cuanto al desarrollo de la originalidad y el tiempo de aplicación del proyecto, así como la iniciativa estudiantil.

\section{REFERENCIAS BIBLIOGRÁFICAS}

Baltar, R. (2015). Diseño y evaluación en el desarrollo de proyectos. Enlace de Bibliotecas digitales.

Cortés, G. (2020). El Proyecto Productivo como Estrategia Pedagógica para Fortalecer las Competencias en Emprendimiento de 
los Estudiantes de la Media Vocacional del Instituto Técnico de Sabana de Torres [Tesis Magisterial, Universidad Autónoma de Bucaramanga].

Damas, G. y Oliva, J. (2017). Gestión educativa institucional y la satisfacción de los estudiantes en las instituciones educativas de la Ugel $N^{o}$ 01, distrito San Juan de Miraflores, Lima, 2015 [Tesis Doctoral, Universidad Cesar Vallejo].

Mendoza, G. (2013). Proyectos Productivos y el aprendizaje significativo de los alumnos de la especialidad Mecánica de Producción de la Facultad de Tecnología de la Universidad Nacional de Educación Enrique Guzmán y Valle [Tesis Magisterial, Universidad Nacional de Educación Enrique Guzmán y Valle].

Rojas, N. y González, I. (2015). Estrategia de gestión pedagógica directiva para transformar la convivencia en el colegio Eduardo Umaña Luna de la localidad Octava de Kennedy [Tesis doctoral, Universidad Libre].

Sañudo. L. (2015). La Transformación de la gestión educativa. Entre el conflicto y el poder. 